\title{
Conception des ouvrages à la mer de la centrale nucléaire de Guangdong en Chine au laboratoire national d'hydraulique de Chatou
}

\author{
M. Darras, B. Latteux et G. Nicollet
}

EDF-LNH

Le Laboratoire National d'Hydraulique de Chatou (LNH) est un Département de la Direction des Etudes et Recherches (DER) d'Electricité de France. Il a pour mission essentielle de résoudre les problèmes d'EDF relevant de la Mécanique des Fluides et de l'Hydraulique et constitue donc le pôle de développement des moyens d'études d'EDF dans ce domaine. Il met à la disposition des utilisateurs d'autres unités de l'Entreprise, des logiciels de simulation numérique en Mécanique des Fluides, et mène avec d'autres Départements de la DER des actions de développement coordonnées sur ces logiciels. Il conduit également des partenariats pour leur diffusion à l'extérieur de l'entreprise.

Les moyens d'étude mis en œuvre couvrent la gamme complète des logiciels scientifiques, des études expérimentales, soit à but de validation de modélisation mathématique, soit à vocation directement appliquée, et des mesures in situ (en mer et en rivière). Ce triple volet est une garantie du maintien du savoir-faire au plus haut niveau, en même temps que du bon choix de l'outil le mieux adapté pour réaliser une étude. Les études particulièrement complexes ou délicates combinent évidemment ces différentes méthodes d'analyse.

L'activité du LNH se répartit globalement en quatre grands chapitres :

- Des études pour les besoins d'autres Directions ou d'autres Départements d'EDF, dans les domaines des aménagements hydroélectriques, des ouvrages des centrales en bord de mer ou en rivière, des réacteurs nucléaires, des chaudières à combustible fossile, des matériels électriques et des utilisations de l'électricité dans l'industrie ou le bâtiment.

- Des prestations de service sous contrat pour des clients publics ou privés, français ou étrangers, dans tous les domaines de compétence du Laboratoire.

- Le développement et la maintenance de logiciels opérationnels de modélisation en Mécanique des Fluides, utilisés par le Laboratoire pour ses études, mais aussi par d'autres Départements de la DER, d'autres Directions d'EDF, ou d'autres partenaires extérieurs dans le cadre d'une politique de commercialisation de ces logiciels.

- Des recherches et développements situés en amont de ces développements de logiciels et qui concernent notamment les méthodes numériques, la turbulence - qui est présente dans pratiquement tous les écoulements étudiés -, les écoulements diphasiques et réactifs, la sédimentologie, la houle et ses effets sur les ouvrages. En raison de l'existence de liens historiques avec les Services Techniques de l'Administration, certaines activités de développements généraux qui intéressent à la fois EDF et les Services Techniques sont soutenues par ces derniers (Services Techniques des Ministères des Transports, de la Mer et de l'Environnement, dans le cadre d'une convention EDF-Administration). Par ailleurs, un nombre croissant d'actions de recherche se situent dans le cadre de projets bénéficiant d'un soutien de la CEE.

L'activité d'études du LNH est essentiellement tournée vers les besoins nationaux, mais il arrive également que le Laboratoire participe à des projets internationaux. C'est un tel cas, assez exemplaire à nos yeux, qui va être exposé ici : la conception des ouvrages d'eau de la centrale nucléaire de Guangdong en Chine.

\section{CADRE DE L'ÉTUDE}

La Centrale nucléaire de Guangdong est située en bord de mer en République Populaire de Chine, à une cinquantaine de kilomètres au nord-est de Hong-Kong. Elle comporte 2 tranches de 900 MWe. Une Société s'est constituée pour mener à bien ce projet commun à la République Populaire de Chine et aux territoires de Hong-Kong, la « Guangdong Nuclear Power Joint Venture Company " (GNPJVC).

Le contrat signé en 1986 a confié à EDF diverses tâches parmi lesquelles les études d'ingénierie générale du projet, de génie civil de l'îlot nucléaire, des ouvrages maritimes et installations annexes.

La conception des ouvrages à la mer, objet de cet exposé, relève des études d'ingénierie. Ces études, réalisées après l'essentiel du programme nucléaire français, ont bénéficié de tous les développements effectués lors de l'étude des centrales de Gravelines, Flamanville, Paluel, Penly, Le Blayais... En particulier, l'expérience acquise alors a permis d'articuler au mieux les études entre elles, et d'utiliser des outils de modélisation très puissants.

\section{LES OUVRAGES D'EAU DE LA CEN- TRALE : OBJECTIFS}

La centrale (fig. l) est située au fond d'une baie relativement fermée, la baie de Daya, mais reste tout de même 


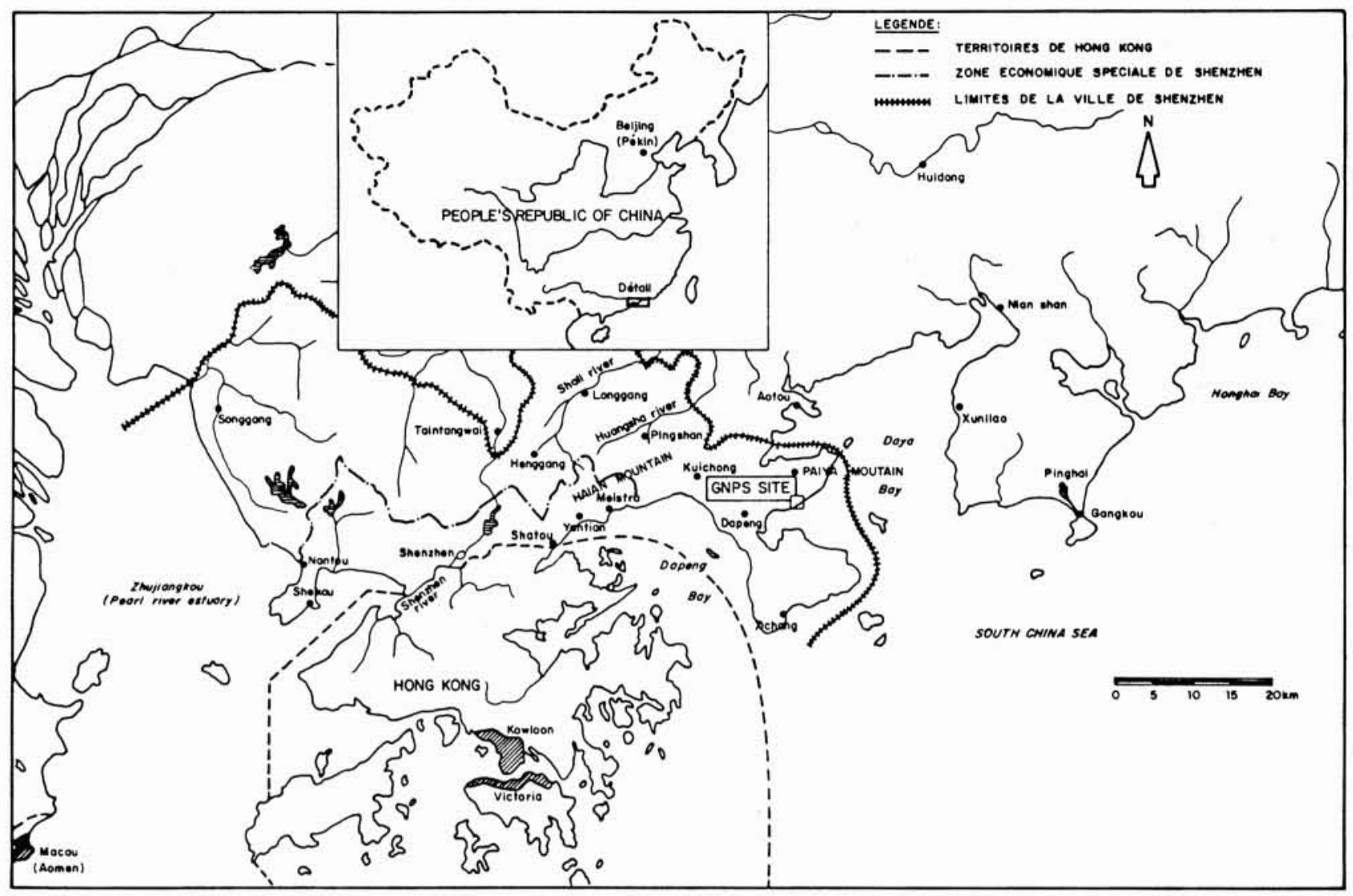

1. Plan de situation de la centrale de Guangdong.

exposée, sur un faible secteur, aux houles générées par les typhons relativement fréquents dans cette région ( 5 à 6 par an en moyenne).

Outre l'alimentation en eau de refroidissement $(95 \%$ pour les deux tranches) et la dilution des eaux échauffées au condenseur, les ouvrages de prise et rejet d'eau de la centrale doivent assurer sa protection contre la houle (amortissement de l'agitation à la prise, protection contre l'inondation par franchissement, etc...) et être dimensionnés en conséquence.

Ces ouvrages sont complétés par une infra-structure portuaire permettant l'accueil des navires $(5,20 \mathrm{~m}$ de tirant d'eau maximum, $120 \mathrm{~m}$ à $160 \mathrm{~m}$ de long), affrêtés pour le transport des équipements lourds de la centrale, fabriqués en Europe.

\section{LE SITE ET SON ENVIRONNE- MENT}

La baie de Daya est une baie peu profonde, $-15 \mathrm{~m}$ par rapport au niveau moyen de la mer à son entrée, $-2 \mathrm{~m}$ à $-6 \mathrm{~m}$ au droit des ouvrages de la centrale.

Si les 2 îlots nucléaires de la centrale sont fondés sur des roches saines, l'altération des terrains croît très rapidement en allant vers la mer. Là, une couche de sable coquillé de 1 à $3 \mathrm{~m}$ d'épaisseur recouvre une couche d'alluvions de 8 à $20 \mathrm{~m}$ d'épaisseur $\left(\gamma_{d}=15 \mathrm{kN} / \mathrm{m}^{3}, C^{\prime}=0, \varphi^{\prime}=40^{\circ}\right)$ dragable à la drague à godets et reposant sur le bed-rock plus ou moins altéré.

Les marées, diurnes ou semi-diurnes, sont d'amplitude relativement faible, environ $3 \mathrm{~m}$. Les courants sont faibles $(0.20 \mathrm{~m} / \mathrm{s}$ au droit du site) et principalement liés à la marée. Cependant, le vent induit des circulations variables au cours du temps, avec des vitesses du même ordre de grandeur que celui des courants de marée.

Une température d'eau de mer très élevée $\left(33^{\circ} \mathrm{C}\right.$ en été) s'ajoute à ces conditions défavorables de courant, et rend le site très peu favorable du point de vue de la dilution thermique. S'il existe une thermocline non négligeable pendant les mois d'été $\left(3^{\circ} \mathrm{C}\right.$ à $\left.5^{\circ} \mathrm{C}\right)$, elle s'est avérée trop instable pour confirmer l'intérêt d'une prise sélective. Cette thermocline semble due à l'influence des fortes pluies de mousson au printemps qui créent une stratification saline, se doublant d'une stratification thermique au cours de l'été.

Par contre, le phénomène de surcote lié aux fortes dépressions créées par les typhons est souvent important ( surcote centennale $=2,88 \mathrm{~m}$ ), d'où un surdimensionnement apparent des ouvrages par rapport au niveau moyen de l'eau. L'état de la mer, modéré tout au long de l'année $\left(H_{s}<2,40 \mathrm{~m}, 95 \%\right.$ du temps) peut devenir très important en cas de typhon (houle centennale : $h_{l} / 10=6,50 \mathrm{~m}, T_{p}=$ $15 \mathrm{~s}$ ), ce qui impose une protection importante des ouvrages. 
Enfin, le transport de sédiments $\left(D_{50}: 0,2 \mathrm{~mm}\right.$ à $\left.0,4 \mathrm{~mm}\right)$ le long de la côte est assez faible (houle et courant faibles la plupart du temps), ce qui limite les risques d'ensablement de la prise d'eau et du port.

\section{LES ÉTUDES DE CONCEPTION DES OUVRAGES À LA MER}

\subsection{Orientations générales du projet}

A partir des données naturelles et de site recueillies par la GNPJVC, EDF a pu définir les critères de conception de la centrale et bâtir le plan de masse autour des principes suivants (fig. 2):

- pour assurer la meilleure dilution possible des eaux chaudes et limiter leur retour vers la prise :

- une prise et un rejet séparés,

- un canal de rejet (plus économique ici qu'un rejet en galeries ou par tuyaux ensouillés compte tenu des conditions géologiques et de la bathymétrie du site) dirigé vers l'Est, séparé de la prise par la digue de protection du port et permettant une mise en vitesse et une orientation du jet vers les courants du centre de la baie, moins faibles qu'au voisinage immédiat du site.

- pour limiter le coût des ouvrages de protection :

- une prise située au sud, à l'intérieur du port, sa sûreté étant assurée par la forme elle-même du chenal de prise et par une double protection contre les bateaux (câbles polyamide) et contre les hydrocarbures et débris divers (barrage flottant).

- prise et port sont protégés par une même digue, perpendiculaire à la direction principale de la houle.

Pour ne pas alourdir les contraintes d'un tel chantier à l'étranger, et compte tenu du planning serré de réalisation notamment pour les ouvrages portuaires, EDF s'est volontairement orientée vers une conception d'ouvrages simples et éprouvés, correspondant aux capacités d'exécution locales :

- digues à talus construites avec les matériaux du site et protégées par des blocs cubiques rainurés, déjà utilisés localement pour la protection de la plate-forme,

- quai en blocs évidés pouvant être placés par voie maritime ou terrestre en reposant sur du ballast.

\subsection{Organisation des études}

Les études engagées ont fait appel à des domaines très différents tels que l'hydraulique, la thermique, le génie civil... De plus, ces études ont une forte imbrication entre elles, toute modification des conclusions de l'une pouvant en remettre en cause une autre. Enfin, les délais étant très serrés, le client chinois a été associé aux décisions prises pas à pas pour éviter des remises en cause en fin de projet.

Pour toutes ces raisons, l'organisation entre les différents participants et l'enchaînement des études (fig. 3) ont été définis d'un commun accord dès le début.

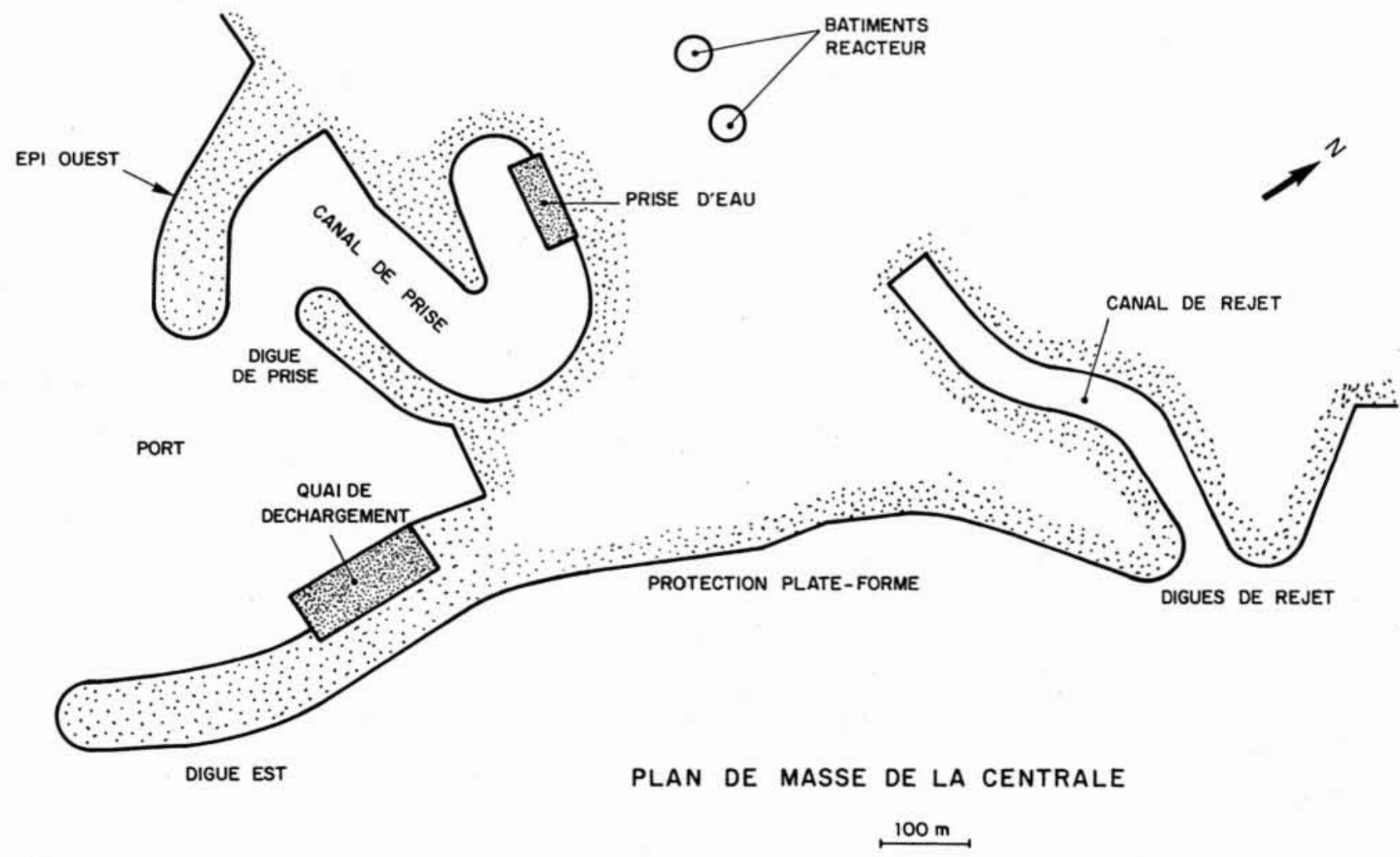

2. Plan de masse de l'usine et des ouvrages de prise et de rejet. 
CRITERES

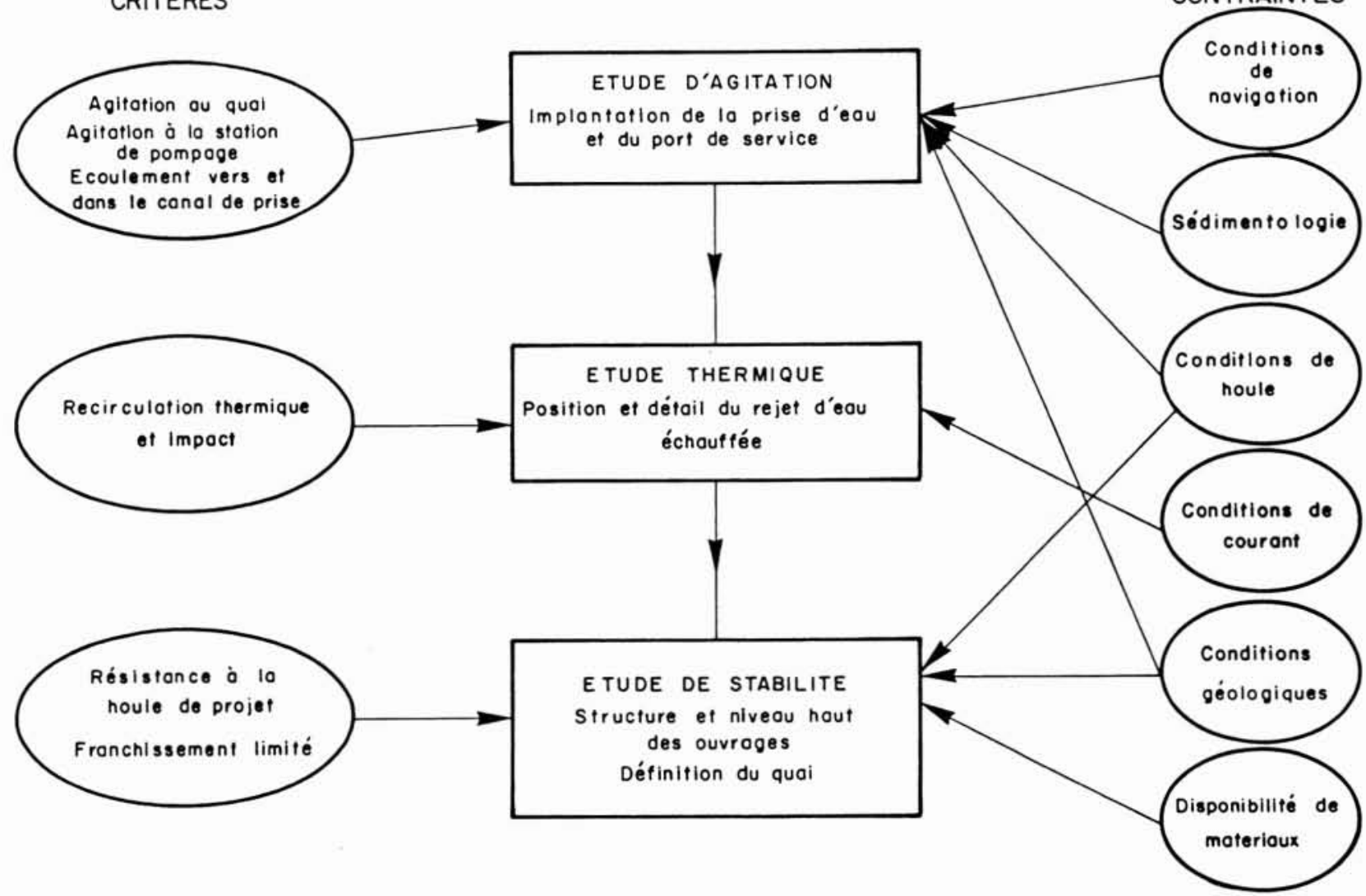

3. Définition des ouvrages à la mer : prise en compte des contraintes et des critères techniques et recherche d'un coût minimal du projet.

La répartition des tâches entre les différents participants fut la suivante :

- EDF/LNH assure les études thermiques et hydrauliques,

- le Port Autonome de Dunkerque (P.A.D.) assure les études du quai de déchargement,

- EDF/Direction de l'Equipement assure l'ingénierie d'ensemble et la coordination avec le client.

Au fur et à mesure, les résultats sont examinés et les répercussions sur l'ensemble des études, discutées entre les participants.

Vis-à-vis du client chinois, des réunions d'avancement périodiques sont organisées et les différentes options prises justifiées pas à pas afin de permettre, autant que faire se peut, un suivi et un accord en ligne du client. De plus, des ingénieurs chinois sont intégrés à différentes équipes du LNH.

L'exécution de l'essentiel des études s'est échelonnée sur un délai d'environ 24 mois. Elles ont représenté pour le LNH trois ans d'ingénieur et sept ans de technicien et ont nécessité l'intervention d'une quarantaine de personnes environ.

\subsection{Description des études}

\subsubsection{Les études d'agitation}

Cette phase du projet avait pour but de définir la position relative de la prise d'eau et du port de service, la localisation du rejet étant faite par la suite après prise en compte des contraintes thermiques. Cette étude a été effectuée par utilisation successive et complémentaire d'un modèle numérique et d'un modèle physique.

Le modèle numérique utilisé est un code du LNH qui permet, pour des conditions données de houle monochromatique incidente, de déterminer la pénétration de la houle à l'intérieur d'un port, en prenant en compte la diffraction derrière une ou deux jetées. L'intérêt d'un tel modèle, relativement simple, est de pouvoir faire une première analyse du problème et de tester rapidement différentes configurations possibles des ouvrages; en outre, l'influence de paramètres tels que la période et la direction de la houle est évaluée aisément. Dans une première phase, deux variantes de port, une solution Nord et une solution Sud, ont été étudiées, cette dernière ayant été proposée par le client à l'instigation d'un laboratoire chinois. Ensuite, après qu'on se soit orienté vers la solution Sud, différentes longueurs et orientations de la digue Est et de l'épi Ouest ont été testées de manière à satisfaire les conditions de projet tout en minimisant la longueur totale de digue afin de réduire le coût du projet.

Dans la solution retenue, la longueur de la digue principale Est a été définie de façon à assurer la protection du port pour les conditions de fonctionnement (le port n'est pas opérationnel en cas de typhons) : la digue Ouest et la digue de prise viennent en complément de la digue Est et assurent la protection de la station de pompage en cas de typhons. 
A l'issue de ces calculs, la meilleure solution obtenue a été testée sur modèle physique. Dans ce but, une maquette du port et de la prise d'eau a été réalisée en similitude de Froude, dans une cuve équipée d'un batteur de houle régulière orientable. L'échelle adoptée était le $1 / 120^{\mathrm{e}}$ (voir photographie en fig. 4). L'agitation résiduelle a été particulièrement étudiée devant le quai de déchargement pour les conditions de service et les conditions de typhons, et devant la station de pompage.

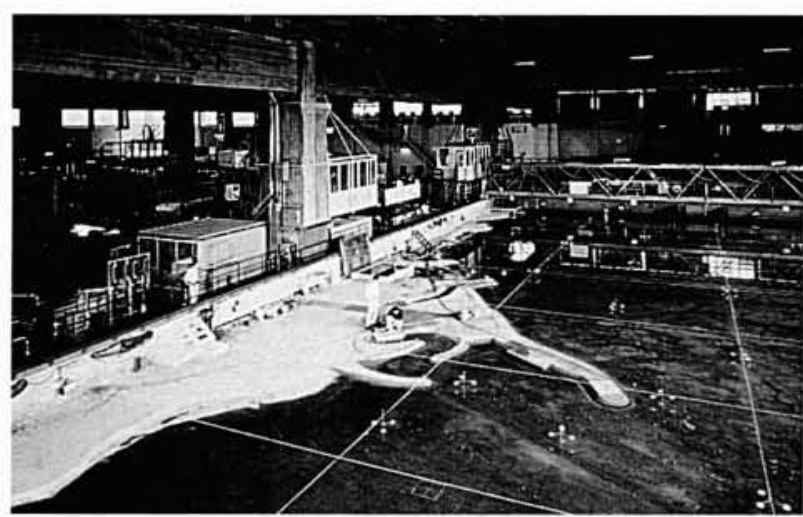

4. Photographie du modèle physique des ouvrages d'eau au $1 / 120^{\mathrm{e}}$.

Par comparaison avec le modèle numérique, les essais ont conduit à infléchir la direction de l'épi Ouest afin de mieux protéger la prise pour les conditions de typhons, et à réduire de $60 \mathrm{~m}$ la longueur de la digue Est.

Enfin, afin de réduire l'agitation à la prise et d'avoir un bon écoulement dans le chenal (suppression des tourbillons et des zones d'eau morte), les pentes des talus du chenal de prise ont été adoucies et la forme d'ensemble modifiée.

\subsubsection{Les études thermiques}

Ces études ont permis de choisir la meilleure configuration de rejet permettant d'assurer une bonne dilution de l'eau de refroidissement de la centrale dans la baie et une recirculation minimale entre le rejet et la prise. Là encore, des modèles numériques et physiques ont été utilisés.

L'impact thermique dans le champ lointain, qui résulte de la dilution de l'eau échauffée sous l'action des courants de marée et des échanges avec l'atmosphère, a été déterminé par utilisation d'un code tridimensionnel d'écoulement. Ces calculs ont été effectués pour différentes conditions de marée ; ils ont montré, d'une part, la forte tendance du panache à rester en surface, et d'autre part, l'effet assez peu marqué de l'éloignement du rejet, à moins d'éloigner celui-ci de la prise de plus de $2500 \mathrm{~m}$ (fig. 5).

L'impact thermique dans le champ proche, c'est-à-dire dans une zone d'environ $1500 \mathrm{~m}$ autour du rejet, a été étudié dans une cuve à courant de $30 \times 50 \mathrm{~m}$. Cette étude, effectuée à l'échelle du $1 / 100^{\circ}$, a permis de quantifier la recirculation directe maximale dans le cas défavorable de courants dus au vent dirigés du rejet vers la prise, et de définir la position et les caractéristiques optimales du rejet (fig. 6 et 7).
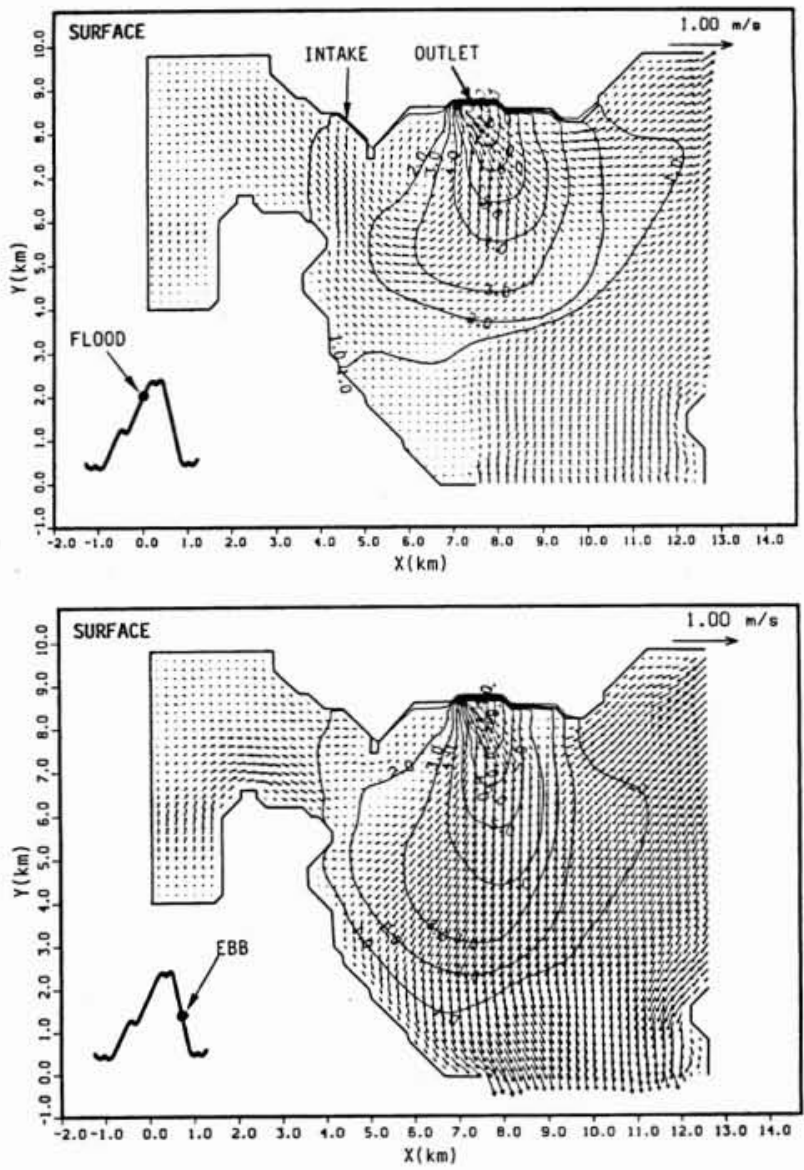

5. Calcul du champ thermique lointain : courant et champs d'échauffements en surface.

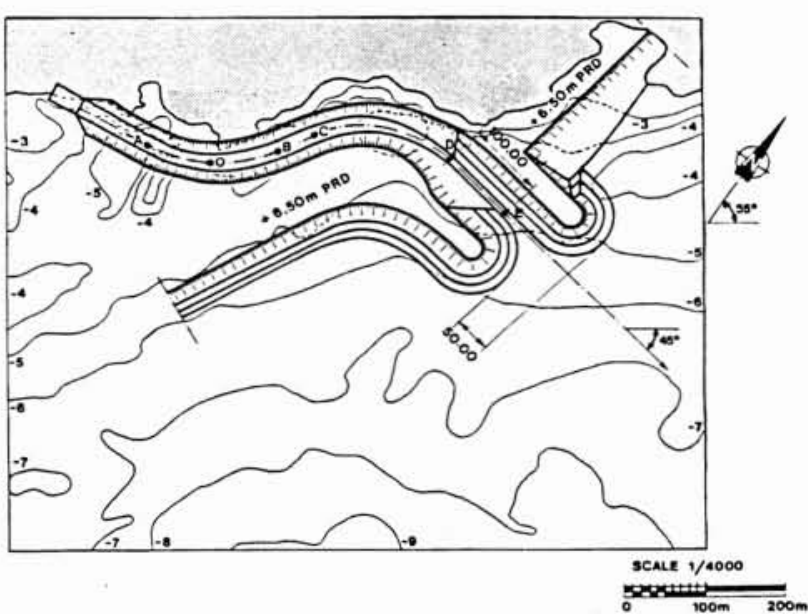

6. Le projet de canal de rejet.

Au vu des résultats des études thermiques, le rejet a finalement été placé en bordure de plate-forme ; l'orientation du canal de rejet est de $45^{\circ}$ par rapport à la côte et la vitesse de rejet de $1 \mathrm{~m} / \mathrm{s}$. La recirculation maximale obtenue est inférieure à $2{ }^{\circ} \mathrm{C}$. 


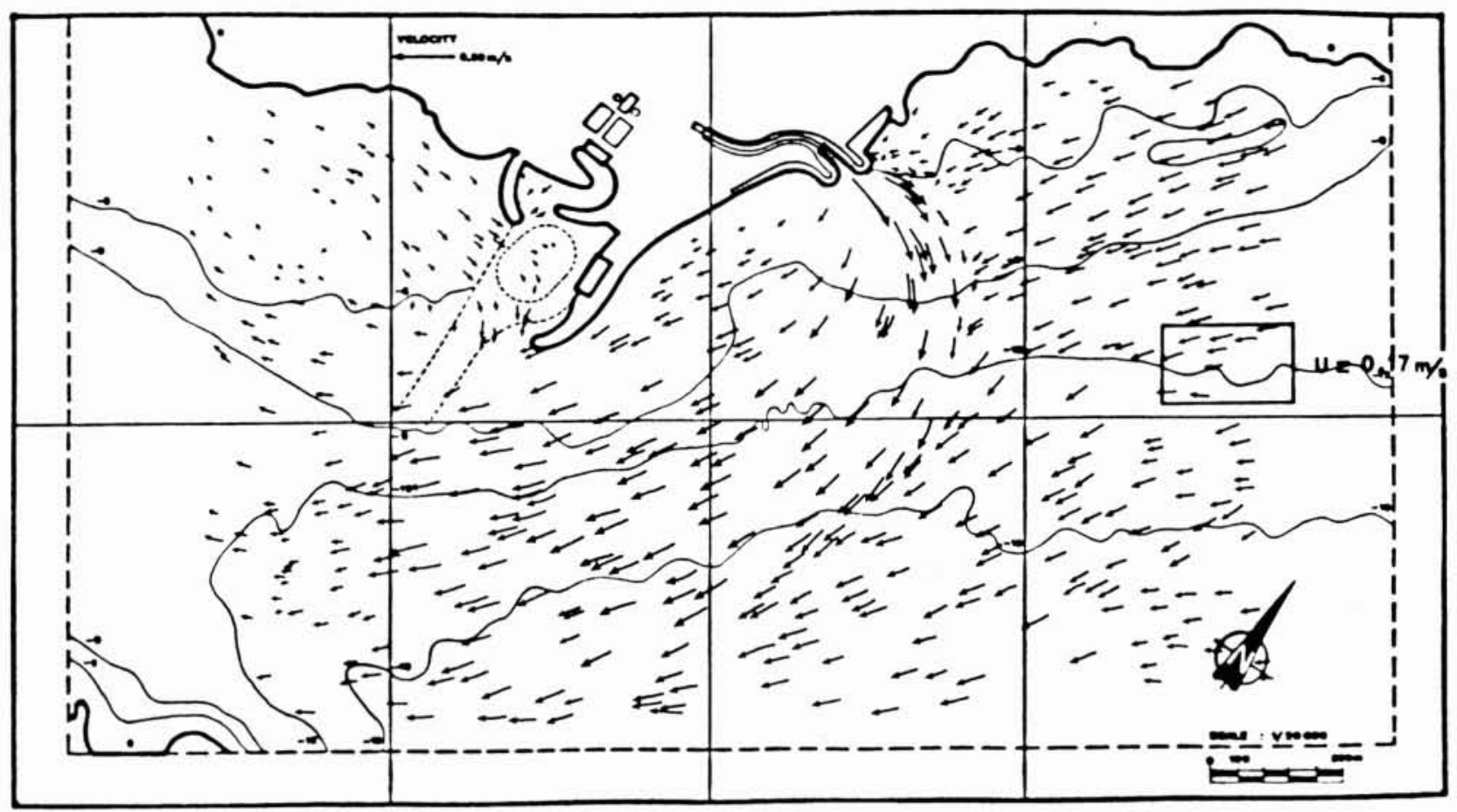

7. Champ de courant de surface sur le modèle réduit.

\subsubsection{Les études de stabilité}

Les études précédentes ayant permis de positionner les différentes parties du projet, la constitution finale des différents ouvrages de protection a été testée sur modèle physique. Ce modèle, construit en similitude de Froude à une échelle de $1 / 66^{\mathrm{c}}$, couvrait une emprise d'environ $3800 \mathrm{~m}$ le long de la côte et de $1500 \mathrm{~m}$ vers le large.

La reproduction de la bathymétrie naturelle a permis de prendre en compte les effets de réfraction de la houle sur le fond et donc de bien représenter les concentrations de houle qui peuvent être dangereuses pour les ouvrages. L'installation utilisée était équipée de générateurs de houle orientables, ce qui permettait de faire varier la provenance de la houle.

La constitution de la variante de départ a été définie à partir des formules théoriques de stabilité et en prenant en compte les contraintes locales de génie civil (matériaux disponibles sur site). Le bloc artificiel adopté est le bloc cubique rainuré qui a déjà été utilisé pour la protection des digues des centrales nucléaires françaises et lors d'une première phase de travaux. Le profil courant initial a été testé et affiné, dans une première étape, dans un canal à houle.

Au cours des essais, plusieurs points intéressants sont apparus. Alors que la stabilité du profil courant n'a pas posé de problèmes particuliers (blocs de $17 \mathrm{t}$ ), des instabilités de la butée de pied le long de la digue Est ont été observées. Ce phénomène peut s'expliquer par le fait que la digue est implantée en relativement faible profondeur d'eau et que donc la butée est située dans une zone très exposée au déferlement de la houle; cet effet est en plus renforcé par des concentrations locales et des incidences de houle obliques causées par des effets bathymétriques. Après plusieurs variantes infructueuses en enrochements naturels de $6-8 \mathrm{t}$, nous avons proposé une butée de pied ensouillée en blocs cubiques qui s'est avérée stable (fig. 8).

Les musoirs des digues de rejet ont également nécessité une attention particulière, l'attaque de vagues plongeant sur les côtés du musoir causant l'expulsion des blocs de carapace. Afin de stabiliser l'ensemble, il a été nécessaire d'augmenter le poids des blocs jusqu'à $44 \mathrm{t}$.

Les hauteurs de calage des différentes digues ont également été déterminées et optimisées sur le modèle : cela a conduit à proposer des hauteurs différentes pour chaque ouvrage en fonction du rôle attribué à chaque digue.

Le modèle a aussi été utilisé pour étudier les phénomènes d'érosion en pied d'ouvrages, car les digues sont érigées sur une zone sableuse. Pour cela, quelques essais à fonds mobiles ont été effectués en représentant le sable naturel par de la bakélite; ils ont permis de définir des tapis d'enrochements à mettre en place devant les ouvrages afin d'éviter l'érosion.

\section{D PROBLÈMES RENCONTRÉS}

Outre les problèmes d'imbrication d'études déjà évoqués, les principaux problèmes rencontrés sont de deux ordres différents :

- l'un, technique, lié aux caractéristiques du site de Daya Bay,

- l'autre relationnel avec le client. 


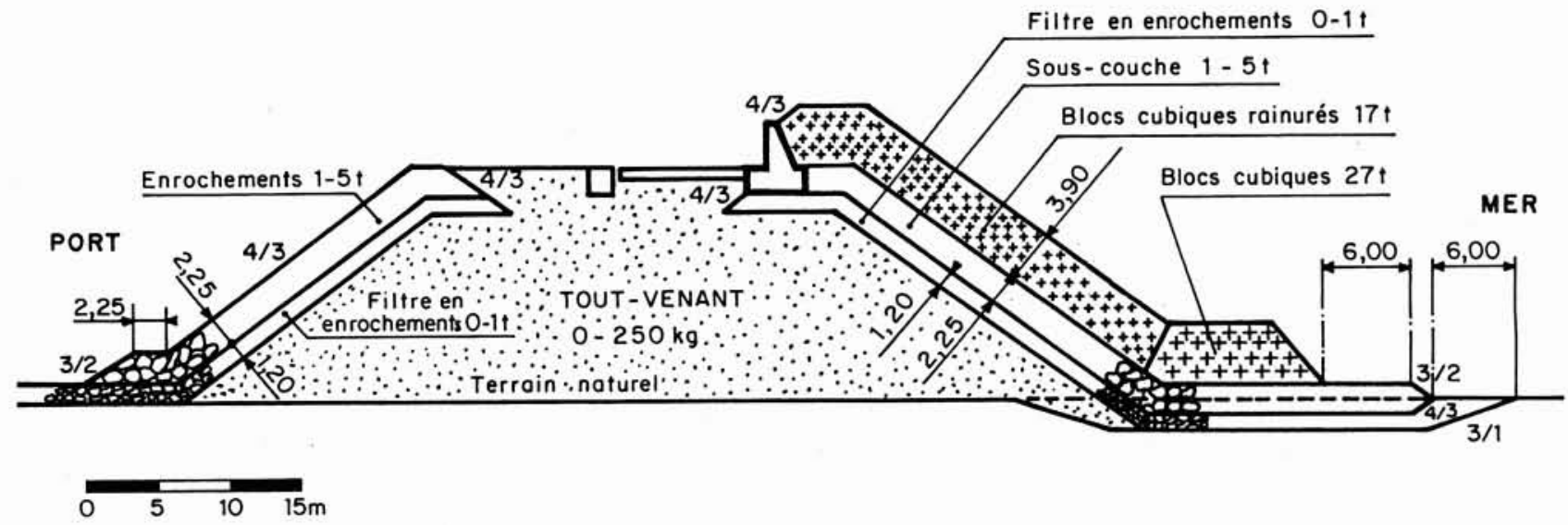

8. Profil courant de la digue Est avec butée ensouillée en blocs cubiques.

\subsection{Problèmes techniques}

Le premier problème technique est lié à l'implantation de la centrale dans une baie semi-ouverte soumise à de faibles courants et de faibles marées. D'où la difficulté théorique d'appréhender les phénomènes hydrauliques résultants et d'évaluer avec précision la recirculation globale vers la prise d'eau du rejet d'eau chaude, ceci malgré des outils mathématiques très élaborés.

Le deuxième problème, ou particularité du site influençant le projet, est l'écart important existant entre les conditions normales et les conditions exceptionnelles à prendre en compte, même très peu fréquentes, par exemple :

- niveau de marée haute (marée faible) et niveau haut extrême (surcote exceptionnelle importante),

- houle courante (faible) et houle exceptionnelle lors d'un typhon (forte).

\subsection{Problèmes relationnels}

Outre le fait que les services d'EDF avaient au départ plus une psychologie de client que de fournisseur, les relations avec le client chinois ont eu à souffrir de difficultés difficilement contournables :

- tout d'abord, l'éloignement du client a rendu les relations assez formelles malgré la présence en France de ses représentants ; d'où parfois des incompréhensions mutuelles, des délais de décision difficilement compatibles avec le planning imposé au bureau d'études,
- un contexte chinois, évidemment différent du contexte français, avec ses techniques propres qu'il faut assimiler pour pouvoir, si c'est le cas, présenter une solution meilleure, de pratique française, qu'il faudra largement justifier (exemples : digue à paroi lisse - dolos - méthodes de construction maritime),

- difficultés à obtenir des données de site suffisantes et fiables,

- un contrat très tendu en devis et en délais ne laissant guère la place à l'étude de nombreuses variantes ni à une optimisation générale des ouvrages malgré un client exigeant sur ce point.

\section{CONCLUSION}

Cette étude, dans un contexte de relations complexes et délicates, a été menée à bien dans les stricts délais imposés grâce à une bonne organisation. En effet, les études engagées faisaient appel à des domaines différents tels que l'hydraulique, la thermique, le génie civil et elles étaient fortement imbriquées.

L'atout primordial de l'ingénierie française était, dans cette occasion, l'expérience des réalisations du programme électro-nucléaire français qui avait permis le développement d'outils et la mise en place d'une organisation efficace.

Le Laboratoire National d'Hydraulique de Chatou a ainsi montré qu'il était toujours à la hauteur de sa réputation bientôt cinquantenaire. 